\title{
Acute idiopathic pericarditis with transient constriction
}

\author{
Andrea Barbieri*, Teresa Grimaldi, Francesca Bursi, Carlo Ratti, \\ Andrea Bellone, Maria Grazia Modena \\ Modena and Reggio Emilia University, Institute of Cardiology, Policlinico Hospital, Via del Pozzo 71, 1100 Modena, Italy
}

Received 22 April 2003; accepted 9 June 2003

Keywords: Acute idiopathic pericarditis; Transient constriction; Eupnoic

\section{Introduction}

The etiology and the clinical pattern of acute pericarditis change frequently and some classic assumption and descriptions are outdated. We report on a case of transient constrictive pericarditis in a healthy young man.

\section{Case report}

A 28-year-old man was referred to a hospital because of acute illness, fever, malaise, and retrosternal chest pain. On the admission, he was eupnoic, the heart rate was 90 beats/min, and the blood pressure was $110 / 80 \mathrm{~mm} \mathrm{Hg}$. Chest roentgenographic findings were unremarkable, and electrocardiography showed near-ubiquitous ST segment (J point) elevations, diffuse PR segment depression and specular elevation in the aVR lead. On auscultation, no pericardial rub was evident. The following laboratory data were obtained: hemoglobin, $13.5 \mathrm{~g} / \mathrm{dl}$, leucocite count, $11 \times 10^{3} / \mathrm{mm}^{3}$, erythrocyte sedimentation rate, $98 \mathrm{~mm}$ in 1 h. Echocardiography disclosed a large pericardial effusion (Fig. 1). The clinical features of active acute pericardial disease subsided with bed rest and nonsteroidal antiinflammatory drugs (ibuprofen $800 \mathrm{mg}$ every $8 \mathrm{~h}$ ). A specific cause was not apparent after assessment according to our internal diagnostic protocol [1]. Acute idiopathic pericarditis was diagnosed. Six days after, his conditions deteriorated: dyspnea, hypotension, and marked giugular venous distension were noted. The echocardio-

\footnotetext{
* Corresponding author. Tel.: +39-339-8260937; fax: +39-59-224223.

E-mail address: olmoberg@libero.it (A. Barbieri).
}

graphic features of effusion disappeared, the pericardium was thickened and the ventricular cavity size varied considerably during respiration. Conventional Doppler [2] and tissue Doppler imaging [3] echocardiographic features typical of constrictive pericarditis became evident (Figs. 1 and 2). During the following 3 weeks, the clinical and echocardiographic signs of pericardial constriction gradually resolved. At follow-up examination 1 and 6 month later, no pathological clinical and echocardiographic findings were detected (Fig. 2).

\section{Discussion}

The evolution to cardiac constriction is a rare occurrence in acute idiopathic pericarditis as compared with other causes of pericardial disease [4]. However, once constriction is detected, it has invariably been considered an irreversible condition regardless of its cause and surgical treatment is required to relieve the symptoms. Transient constrictive pericarditis was first reported in 1987 [5]. The investigators described the three phases of this clinical entity. During the initial phase, a moderate to large amount of circumferential pericardial effusion was noted, and pericardiocentesis was necessary in some patients. In phase II, anti-inflammatory treatment yielded clinical improvement and diminished pericardial effusion; however, at a mean interval of 11 days (range, 5-30) after the detection of pericardial effusion, clinical signs and/or laboratory findings typical of constriction occurred. During the phase of constriction, the coexistence of a small amount of pericardial fluid was possible. In phase III (normalization), these constrictive changes returned to normal at a mean of 2.7 months (range, 12 


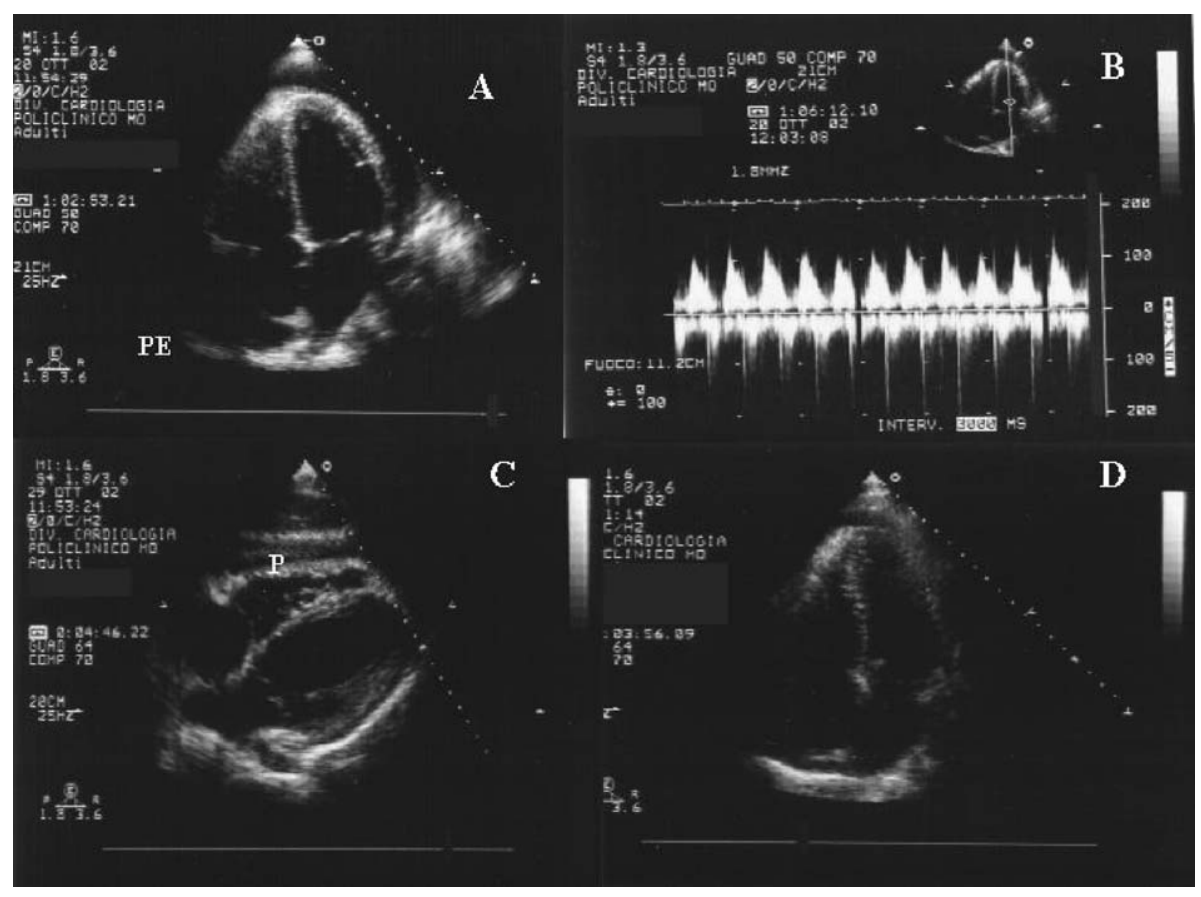

Fig. 1. A) Phase I. Initial B-mode echocardiogram, demonstrating moderate-sized pericardial effusion (PE) and findings suggestive of elevation of intrapericardial pressure (right atrial chamber collapse, arrows). B) Pulsed-wave Doppler study of mitral valve velocities showing no variation in early rapid filling during respiration. C) Phase II. Repeated B-mode echocardiogram examination after 9 days of medical treatment. Pericardial effusion has reduced and organized, pericardium $(\mathrm{P})$ has thickened $(10 \mathrm{~mm})$. D) Ventricular septal motion is abnormal, shifting toward LV during inspiration (arrow).

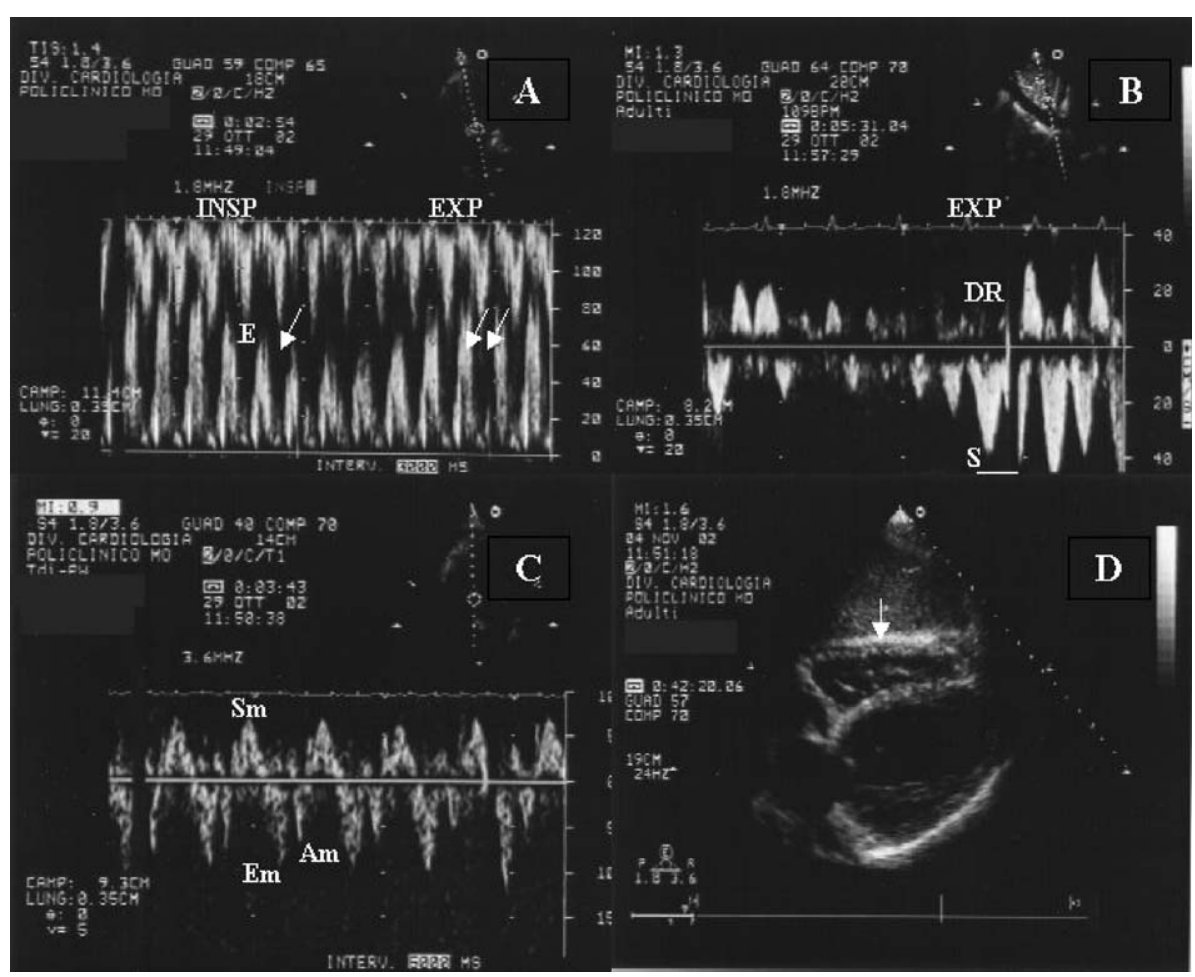

Fig. 2. A) Pulsed-wave Doppler recordings mitral inflow velocities obtained by placing sample volume between leaflet tips during diastole. Note pronunciated decrease in velocity of early (E) rapid filling (single arrow) after onset of inspiration (INSP) and increase (double arrow) during expiration (EXP). B) Hepatic vein Doppler velocities, showing diastolic flow reversal (DR) with expiration (EXP). $D=$ diastolic forward flow; EXP=expiration; $S=$ systolic forward flow. $C$ ) Mitral annular tissue Doppler demonstrating normal axial velocities. Sm=Systolic, Em=early diastolic, Am=atrial contraction velocities. D) Phase III. After 6 days of treatment with hydrocortisone, pericardial effusion has disappeared, pericardial thickness decreased substantially (arrow), and patient had relief of symptoms. 
days to 10 months) after pericardial constriction was diagnosed, and all patients were reported free of constriction at a mean follow-up of 31 months. The mechanism of these findings would be a transiently thickened pericardium (as a consequence of edema, fibrin deposition or inflammation) that would return to normal [6]. In some patients, these phases may be short-lived and may not be noted unless the clinician is aware of this possible evolution, but in other cases the constriction phase may last for several months and lead to management dilemmas.

\section{Conclusion}

Constriction can be acute or subacute and transient. Persistent misconception should be acknowledged to avoid diagnostic and therapeutic pitfalls.

\section{References}

[1] Soler-Soler J, Sagristà-Sauleda J, Permanyer-Miralda G. Management of pericardial effusion. Heart 2001;86:235-40.

[2] Hatle LK, Appleton CP, Popp RL. Differentiation of constrictive pericarditis and restrictive cardiomyopathy by Doppler echocardiography. Circulation 1989;79:357-70.

[3] Garcia MJ, Rodriguez L, Ares MA, Griffin BP, Thomas JD, Klein AL. Differentiation of constrictive pericarditis from restrictive cardiomyopathy: assessment of left ventricular diastolic velocities in the longitudinal axis by Doppler tissue imaging. J Am Coll Cardiol 1996;27:108-14.

[4] Permanyer-Miralda G, Sagristà-Sauleda J, Soler-Soler J. Primary acute pericardial disease: a prospective series of 231 consecutive patients. Am J Cardiol 1985;56:623-30.

[5] Sagristà-Sauleda J, Permanyer-Miralda G, Candell-Riera J, Angel J, Soler-Soler J. Transient cardiac constriction: an unrecognized pattern of evolution in effusive acute idiopathic pericarditis. Am J Cardiol 1987;59:961-6.

[6] Oh JK, Hatle LK, Mulvagh SL, Tajik J. Transient constrictive pericarditis: diagnosis by two-dimensional Doppler echocardiography. Mayo Clin Proc 1993;68:1158-11164. 